\title{
Romantismes, l'esthétisme en acte, sous la direction de Jean-Louis Cabanès
}

\section{Esther Pinon}

\section{(2) OpenEdition}

1 Journals

\section{Édition électronique}

URL : https://journals.openedition.org/studifrancesi/3116

DOI : 10.4000/studifrancesi.3116

ISSN : 2427-5856

Éditeur

Rosenberg \& Sellier

\section{Édition imprimée}

Date de publication : 1 juillet 2013

Pagination : 461-462

ISSN : 0039-2944

\section{Référence électronique}

Esther Pinon, «Romantismes, l'esthétisme en acte, sous la direction de Jean-Louis Cabanès », Studi Francesi [En ligne], 170 (LVII | II) | 2013, mis en ligne le 30 novembre 2015, consulté le 02 février 2023. URL : http://journals.openedition.org/studifrancesi/3116; DOI : https://doi.org/10.4000/studifrancesi. 3116

Ce document a été généré automatiquement le 2 février 2023.

\section{(c) (†)}

Creative Commons - Attribution - Pas d'Utilisation Commerciale - Pas de Modification 4.0 International - CC BY-NC-ND 4.0

https://creativecommons.org/licenses/by-nc-nd/4.0/ 


\title{
Romantismes, l'esthétisme en acte, sous la direction de Jean-Louis Cabanès
}

\author{
Esther Pinon
}

\section{RÉFÉRENCE}

AA. VV., Romantismes, l'esthétisme en acte, sous la direction de Jean-Louis CABANÈs, Nanterre, Presses Universitaires de Paris Ouest, 2009, «Orbis litterarum», pp. 357.

1 Ce volume regroupe les actes du deuxième congrès international de la Société des Études romantiques et dix-neuviémistes, portant sur «L'esthétique en acte». Il offre un panorama vaste et approfondi de l'irruption de l'esthétique dans le champ littéraire français à l'époque romantique. Mais, le titre le suggère et l'introduction le confirme, les auteurs ne se proposent pas seulement d'étudier l'esthétique comme une discipline théorique et prescriptive, ils s'attachent surtout à l'examen d'un faire, et décèlent dans les textes, fictifs ou non, une pensée parfois explicite, parfois sous-jacente, du beau ou du sublime.

2 L'introduction de Philippe HAMON (pp. 13-25) qui, en explorant les diverses implications du titre retenu, réalise une traversée de la première moitié du XIX $x^{e}$ siècle, est prolongée par deux études qui adoptent également une perspective diachronique: José-Luis DIAZ (De la poétique à l'esthétique (1800-1850), pp. 27-47) montre comment, malgré des résistances persistantes, l'esthétique se substitue progressivement aux poétiques, fortement discréditées, et suggère les conséquences de cette mutation étroitement liée à la promotion de «l'Art». Quant à Élisabeth DECULTOT (Le débat français sur l'esthétique philosophique allemande (1820-1850), pp. 49-63), elle examine la réception française d'une discipline étrangère qui, si elle tend à s'imposer au cours de la période étudiée, n'en demeure pas moins l'objet de réticences fortes et révélatrices de la rencontre complexe de deux cultures.

3 La section suivante retrace les mutations et les permanences de l'esthétique des Lumières au romantisme, et des théories aux réalisations: Mildred GALLAND-SZYMKOWIAK 
(L'esthétique comme unité du théorique et du pratique dans le "Système transcendantal" de Schelling, pp. 67-81) et René-Marc PILLE (L'esthétique selon Schiller. Prolégomènes à l'étude de sa réception, pp. 83-94) rappellent l'apport majeur de la pensée allemande dans la constitution d'un esthétisme européen à l'aube du xix siècle, mais révèlent aussi sa diversité et ses variations subtiles. L'article de Laurent CLAUZADE (Le réalisme esthétique de la philosophie spiritualiste, une esthétique de l'idéal, pp. 95-107) signale quant à lui que l'Angleterre joue aussi son rôle dans ce vaste mouvement européen, en interrogeant l'influence sur Théodore Jouffroy de la théorie du sens commun de Thomas Reid. Les études de Dominique PEYRACHE-LEBORGNE (Hugo, le grotesque et l'arabesque, pp. 109-122) et Baldine SAINT-GIRONS (Esthétique, sublime et monstrueux, pp. 123-140) interrogent, eux, la circulation de motifs esthétiques dans l'Europe romantique. La première retrouve les racines du grotesque hugolien dans l'esthétique de l'arabesque, perceptible notamment chez les frères Schlegel; la seconde montre l'articulation du sublime et du monstrueux en comparant le Frankenstein de Mary Shelley, le Sombreval de Barbey d'Aurevilly et le Dr Jekyll de Stevenson.

4 L'article que Stéphanie tRIBOUILLARD consacre à Mme de Staël ( $Y$ a-t-il une esthétique staëlienne en 1800?, pp. 155-170) poursuit cette exploration interculturelle de l'esthétisme naissant, en montrant le rôle fondateur d'une pensée des esthétiques nationales en germe dans De la littérature, et qui trouve son plein épanouissement dans De l'Allemagne. Mais cette étude s'inscrit aussi dans une réflexion sur la place de l'esthétique - ou des esthétiques - chez les précurseurs du romantisme français, réflexion que viennent compléter les articles de Gérard GENGEMBRE (Bonald ou l'esthétique sociale de la littérature, pp. 143-154), de Béatrice DIDIER (Le beau idéal selon Chateaubriand, pp. 171-181) et de Jean-François JEANDILlou (L'esthétique de la langue selon Nodier, pp. 183-195). À travers les relectures des quatre essayistes se dessine un rapport complexe du premier romantisme à la pensée classique du beau, qui imprègne profondément et durablement les esprits, mais se trouve renouvelé en profondeur.

Ce sont ensuite des approches collectives du beau qui sont examinées, dans une section intitulée "Sociabilités au cours du siècle», qui montre à quel point le développement d'une esthétique peut être conditionné par des choix éditoriaux. Les articles de Celina MOREIRA DE MELLO ("L'Artiste" (1831-1838): l'artiste, les Salons et la critique d'art, pp. 199-211) et d'Anthony GLINOER (À la lisière de l'avant-garde esthétique: «La Liberté, journal des arts», pp. 213-226) montrent comment une ligne éditoriale peut renouveler la conception du rapport entre les arts, ou inventer un militantisme esthétique; François KERLOUÉGAN (Les manuels de beauté romantiques: une esthétique mise en pratique, pp. 227-240) décèle les implications idéologiques du discours esthétique tenu dans les manuels de beauté; quant à Françoise SYLVOS (Le quête du beau dans les utopies de la première moitié du XIXe siècle, pp. 241-255), elle montre que les essais mais aussi les fictions utopiques articulent l'esthétique et le social.

6 Enfin, la dernière section de l'ouvrage met au jour certains principes esthétiques propres aux artistes romantiques et révélés par leurs œuvres. L'article de MarcMathieu MÜNCH, qui s'attache à redéfinir l'ironie romantique (Ironie romantique et esthétique, pp. 259-268), trouve un prolongement dans l'évocation du cas particulier de Heine, dont l'ironie et la pensée du «surnaturalisme» irriguent la critique d'art et la réflexion sur les esthétiques nationales (Jean LACOSTE, Heinrich Heine et le romantisme français: ironie allemande et romantisme français, pp. 269-279). Timothée PICARD se penche lui aussi sur le romantisme allemand en analysant le rôle fondateur d'Hoffmann dans 
l'irruption d'une esthétique musicale en littérature (L'esthétique musicale, d'Hoffmann à ses héritiers, pp. 281-290). Les articles suivants en revanche sont centrés sur les romantismes français: Brigitte DIAZ étudie la pensée du beau et des arts que suggèrent les Lettres d'un voyageur (Sand et la "fraternité des arts», pp. 291-309), Christèle COULEAUMAIXENT s'interroge sur la tension qui s'établit chez Balzac entre esthétique en acte, représentations de l'artiste et interventions auctoriales («Suivez le guide!». Le commentaire esthétique du romanesque dans le récit balzacien, pp. 311-325), Paolo TORTONESE révèle les contradictions inhérentes à l'anti-utilitarisme de Théophile Gautier pour mieux en monter la fécondité artistique (À quoi cela sert-il? La polémique contre l'utilitarisme dans la préface de "Mademoiselle de Maupin", pp. 325-333), Jean-Philippe снімот, en confrontant les écrits et les toiles de Delacroix, évoque l'invention d'un «non-discours» esthétique (Delacroix peintre et critique: questions au silence, pp. 335-346) et Michèle HANNOOSH démontre la prégnance de la pensée esthétique et de l'influence picturale sur l'œuvre de Michelet (Imagination esthétique et conscience historique: Jules Michelet et les arts plastiques, pp. 347-357).

7 On peut regretter la présence de quelques fautes dans un volume par ailleurs remarquable, qui réunit des études toujours complémentaires et jamais redondantes, et offre une réflexion particulièrement stimulante. 